\title{
Spin Manipulation with an RF Wien-Filter at COSY
}

\author{
Sebastian Mey ${ }^{* \dagger}$ \\ Institut für Kernphysik, Forschungszentrum Jülich GmbH, 52425 Jülich, Germany \\ III. Physikalisches Institut B, RWTH Aachen, Otto-Blumenthal-Str., 52074 Aachen, \\ Germany \\ E-mail: s.meyefz-juelich.de

\section{Ralf Gebel} \\ Institut für Kernphysik, Forschungszentrum Jülich GmbH, 52425 Jülich, Germany \\ E-mail: r.gebelefz-juelich.de
}

\begin{abstract}
The JEDI Collaboration (Jülich Electric Dipole Moment Investigations) is developing tools for the measurement of permanent EDMs (Electric Dipole Moments) of charged, light hadrons in dedicated storage rings. The Standard Model predicts unobservably small magnitudes for these EDMs. A non-vanishing value due to CP violating sources beyond the Standard Model may be detected by measuring a tiny vertical polarization buildup in a beforehand horizontally polarized beam. This technique requires a spin tune modulation by an RF Dipole without inducing any coherent beam oscillations.

In the course of 2014, a prototype RF (Radio-Frequency) ExB-Dipole has been successfully commissioned and tested at COSY (the Cooler Synchrotron in Jülich, Germany). The force of a radial magnetic field is canceled out by a vertical electric one. In this configuration, the dipole fields form a Wien-Filter that directly rotates the particles' polarization vector and thus allows the determination of the beam and spin dynamics behavior of such a device. We verified that the RF Dipole can be used to continuously rotate the vertical polarization vector of a $970 \mathrm{MeV} / \mathrm{c}$ deuteron beam without exciting any coherent beam oscillations.
\end{abstract}

XVIth International Workshop in Polarized Sources, Targets, and Polarimetry, PSTP2015,

14-18 September 2015,

Bochum, Germany

\footnotetext{
*Speaker.

${ }^{\dagger}$ Contribution on behalf of the JEDI Collaboration.
} 


\section{Motivation}

The motion of a relativistic particle's spin in an static, electromagnetic storage ring $^{1}$ with non vanishing EDM contributions is given by the generalized Thomas-BMT Equation [1, 2]:

$$
\begin{aligned}
\frac{\mathrm{d} \vec{S}}{\mathrm{~d} t}=\vec{S} \times\left(\vec{\Omega}_{\mathrm{MDM}}+\vec{\Omega}_{\mathrm{EDM}}\right), \quad \vec{\Omega}_{\mathrm{MDM}} & =\frac{q}{\gamma m}\left((1+\gamma G) \vec{B}-\left(\frac{\gamma}{\gamma+1}+\gamma G\right) \vec{\beta} \times \frac{\vec{E}}{c}\right) \\
\vec{\Omega}_{\mathrm{EDM}} & =\frac{q}{m} \frac{\eta}{2}\left(\frac{\vec{E}}{c}+\vec{\beta} \times \vec{B}\right) .
\end{aligned}
$$

Here, the anomalous magnetic moment $G$ is given by the particle's $\mathrm{MDM}^{2}, \vec{\mu}=2(G+1) \frac{q}{2 m} \vec{S}$. This corresponds to $G=-0.142$ for deuterons. As an analogue, the dimensionless factor $\eta$ describes the strength of the particle's permanent EDM. A Standard Model prediction for light hadrons of $d=\eta \frac{q}{2 m c} \vec{S} \approx 10^{-32}$ ecm gives $\eta \approx 10^{-16}$.

In a purely magnetic storage ring, all the terms containing electric fields in Eq. 1.1 vanish and the EDM contribution due to the interaction with the motional electric field $(\vec{\beta} \times \vec{B})$ will lead to a tiny tilt of the spin's precession axis. This leads to an oscillation of the vertical polarization component, but for $\eta \approx 10^{-16}$ its contribution is far below measurable. Adding an RF Wien-Filter with a vertical magnetic and radial electric field oscillating on a harmonic of the spin precession to the ring's lattice introduces an artificial spin resonance that, in combination with the EDM's interaction with the motional electric field in the rest of the ring, results in a constant in time part of the horizontal spin precession. The beam motion is ideally not disturbed due to the Lorentz force cancellation. Now the motional electric field in the ring can generate a constant EDM torque which in turn leads to a continuous buildup of vertical polarization in a beforehand horizontally polarized beam [3].

\section{Setup of the Prototype}

While the above described approach could provide a measurable EDM signal, it doesn't provide an observable to characterize the RF Wien-Filter itself. Therefore, a first prototype with a radial magnetic field, $\vec{B}=\left(\hat{B}_{x}, 0,0\right)^{T}$, and a vertical electric field, $\vec{E}=\left(0, \hat{E}_{y}, 0\right)^{T}$, has been commissioned. The RF field combination introduces an artificial spin resonance which directly rotates the beam polarization vector.

Expressing the electric field in Eq. 1.1 in terms of the magnetic field using the Lorentz force compensation leads to a simple formula for the spin precession in an ideal Wien-Filter [4]. Also, the spin precession due to the particles' EDM is zero in case of cancelled Lorentz force:

$$
\begin{aligned}
\frac{\vec{E}}{c}=-\vec{\beta} \times \vec{B} \Rightarrow \quad \vec{\Omega}_{\mathrm{MDM}} & =\frac{q}{\gamma m}\left((1+\gamma G) \vec{B}-\left(\gamma G+\frac{\gamma}{\gamma+1}\right) \beta^{2} \vec{B}\right)=\frac{q}{\gamma m} \frac{1+G}{\gamma} \vec{B} \\
\vec{\Omega}_{\mathrm{EDM}} & =\overrightarrow{0} .
\end{aligned}
$$

\footnotetext{
${ }^{1}$ neglecting longitudinal field components, $\vec{\beta} \cdot \vec{B}=\vec{\beta} \cdot \vec{E}=0$

${ }^{2}$ Magnetic Dipole Moment
} 


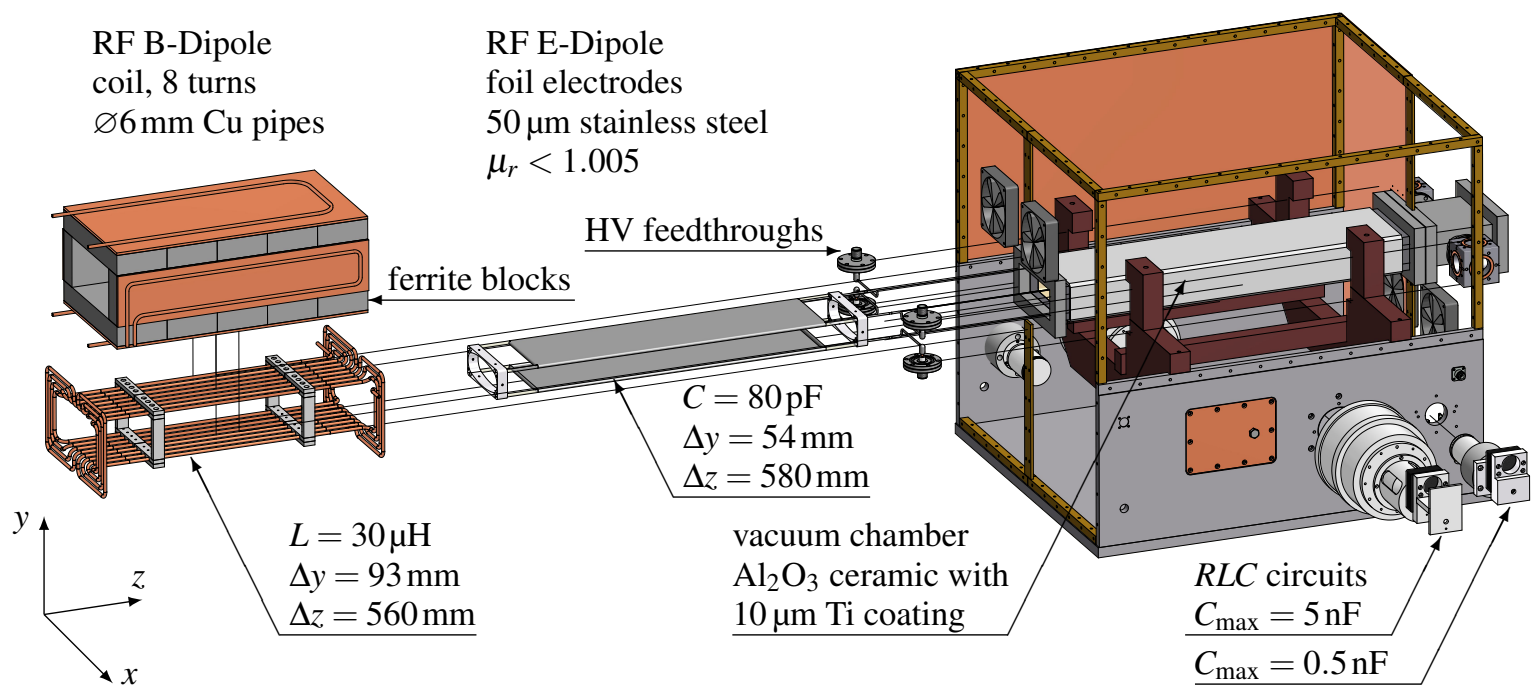

Figure 1: An explosion drawing showing the main components of the RF ExB-Dipole.

The particles sample the localized RF field distributions once every turn. Their contribution may be approximated by the integrated field along the particles' path assigned to a point-like device at an orbital angle $\theta$ :

$$
b(\theta)=\int \hat{B}_{x} \mathrm{~d} l \cos \left(\frac{f_{\mathrm{RF}}}{f_{\mathrm{rev}}} \boldsymbol{\theta}+\phi\right) \sum_{n=-\infty}^{\infty} \delta(\theta-2 \pi n) .
$$

The resonance strength $\left|\varepsilon_{K}\right|$ of such a device is given by the amount of spin rotation in the device per turn and can be calculated by the Fourier integral over one turn $[5,6]$ :

$$
\left|\varepsilon_{K}\right|=\frac{\Omega_{\mathrm{spin}}}{\Omega_{\mathrm{rev}}}=\frac{1+G}{2 \pi \gamma} \oint \frac{b(\theta)}{B \rho} e^{i K \theta} \mathrm{d} \theta=\frac{1+G}{4 \pi \gamma} \frac{\int \hat{B}_{x} \mathrm{~d} l}{B \rho} \sum_{n} e^{ \pm i \phi} \delta\left(n-K \mp \frac{f_{\mathrm{RF}}}{f_{\mathrm{rev}}}\right) .
$$

An artificial spin resonance occurs at side-bands with a frequency corresponding to the spin tune:

$$
K \stackrel{!}{=} \gamma G=n \pm \frac{f_{\mathrm{RF}}}{f_{\mathrm{rev}}} \Leftrightarrow f_{\mathrm{RF}}=f_{\text {rev }}|n-\gamma G|, n \in \mathbb{Z} .
$$

In the scope of the current JEDI experiments, deuterons with a momentum of $970 \mathrm{MeV} / \mathrm{c}$ are stored at COSY [7]. In this case, $\gamma=1.126$ and the resulting spin tune is $\gamma G=-0.1609$. The fundamental mode is located at $f_{\mathrm{RF}}=121 \mathrm{kHz}$ with $n= \pm 1$ harmonics at $629 \mathrm{kHz}$ and $871 \mathrm{kHz}$.

The magnetic dipole field for the desired Wien-Filter field combination is generated by means of a coil wound lengthwise around a ceramic part of the beam-pipe. Ferrite blocks bundle the field lines and flatten the transverse field distribution. To generate an oscillation on the above derived frequencies, the coil is connected to an adjustable, parallel resonance circuit with a quality factor of $Q \approx 20$, housed inside a common RF shielding box. A similar, but separate resonance circuit drives the electric RF Dipole. The electric field is generated by the potential difference between two stainless steel electrodes inside the vacuum chamber spanned over glass rods held by a frame inside the flanges of the ceramic beam-chamber. For details see Fig.1.

Due to water cooling of the coil and fans dissipating the remaining lost power it is possible to run the system up to $90 \mathrm{~W}$ input power in continuous, long term operation. The corresponding operating parameters have been collected in Table 1. 


\begin{tabular}{ccc}
\hline & RF B-Dipole & RF E-Dipole \\
\hline$\hat{U}$ & & $2 \mathrm{kV}$ \\
$\int \hat{E}_{y} \mathrm{~d} l$ & & $24.1 \mathrm{kV}$ \\
$\hat{I}$ & $5 \mathrm{~A}$ & \\
$\int \hat{B}_{x} \mathrm{~d} l$ & $0.175 \mathrm{Tmm}$ & \\
$f_{\mathrm{RF}}$ range & $630 \mathrm{kHz}$ to $1170 \mathrm{kHz}$ & $630 \mathrm{kHz}$ to $1060 \mathrm{kHz}$ \\
\hline
\end{tabular}

Table 1: The RF ExB-Dipole at $90 \mathrm{~W}$ RMS input power.

As Fig. 2 shows, the transverse distribution of the main field components is flat across the center of the beam chamber. But due to different drop-off rates of the electric and magnetic field, particles will encounter a down-up kick at the entrance and a corresponding up-down kick at the exit of the Wien-Filter. The geometry has been optimized insofar that particles with the reference momentum entering the system on axis won't get any vertical excursion, as shown in Fig. 2c. Particles off momentum will probe different Lorentz forces, leading to a slight spread in vertical beam size in the order of $20 \mathrm{~nm}$ for a typical momentum spread of $\Delta p / p=10^{-4}$. The feeds to the coil and the electrodes introduce a slight asymmetry in the field distribution, resulting in a small horizontal kick which gives an offset of $-40 \mathrm{~nm} 1 \mathrm{~m}$ behind the device center. Considering a normalized emittance of $\varepsilon \approx 1 \pi \mathrm{mm}$ mrad for a well cooled deuteron beam at COSY and beta functions calculated with the Mad model of the COSY optics from the May 2015 JEDI beam-time, one gets an estimate for the beam size at the location of the RF ExB-Dipole:

$$
\beta_{x}=4.1 \mathrm{~m} \Rightarrow\langle x\rangle=\sqrt{\varepsilon \beta_{x}} \approx 4 \mathrm{~mm}, \quad \beta_{y}=21.1 \mathrm{~m} \Rightarrow\langle y\rangle=\sqrt{\varepsilon \beta_{y}} \approx 8 \mathrm{~mm} .
$$

The beam disturbance of the RF ExB-Dipole with compensated Lorentz force is 5 orders of magnitude below these beam size estimates. This is well below even detectable as long as the device isn't operated on a resonance of the revolution frequency.

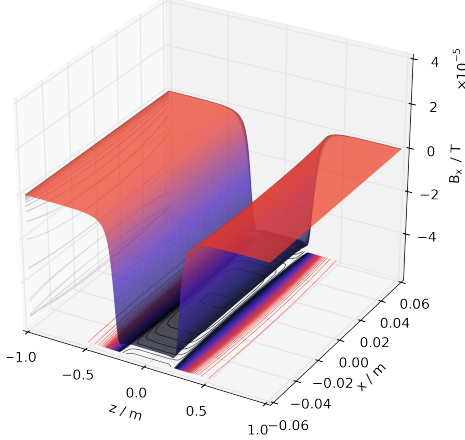

(a) $\hat{B}_{x}$ across the center of the beam-pipe at $y=0.0 \mathrm{~m}$, $\hat{I}_{\text {RF-B }}=1 \mathrm{~A}$.

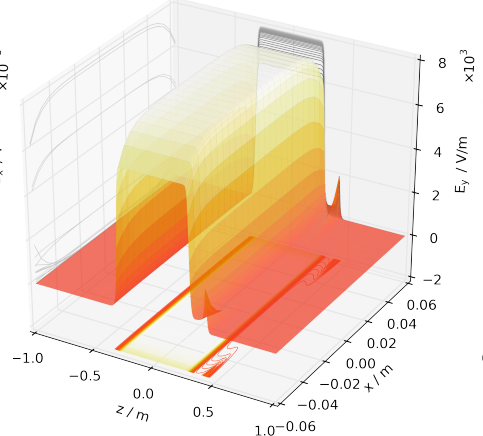

(b) $\hat{E}_{y}$ across the center of the beam-pipe at $y=0.0 \mathrm{~m}$, $\hat{U}_{\mathrm{RF}-\mathrm{E}}=146 \mathrm{~V}$.

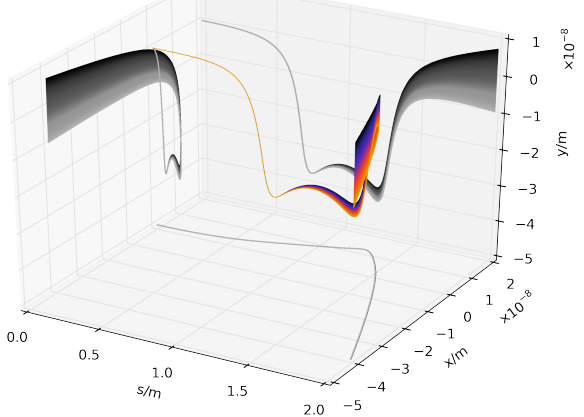

(c) Trajectories of 1001 deuterons, $p=970 \mathrm{MeV} / \mathrm{c}, \Delta p / p=10^{-4}$, $x_{0}=y_{0}=0 \mathrm{~mm}, x_{0}^{\prime}=y_{0}^{\prime}=0 \mathrm{rad}$.

Figure 2: Simulation of the main field components generated by the RF ExB-Dipole. The right panel shows the result of particle tracking along the reference orbit $s$ through these fields. 


\section{Measurements}

For day-to-day operation of the RF system, a beam-based solution for Lorentz-Force cancellation is necessary. The phase as well as the amplitudes of the $E$ and $B$ fields have to be optimized. By modifying the strengths of two of the main quadrupole families in the synchrotron, the fractional vertical betatron tune $q_{y}$ is shifted so that a harmonic of the betatron oscillation frequency coincides exactly with the RF frequency on one of the spin resonance harmonics, (see Eq. 2.4). In combination with a limited acceptance by a carbon block directly above the beam, which serves as target for the polarimeter, this gives maximum sensitivity of beam stability to induced vertical beam oscillations. The COSY beam current transformer can thus be used as a precise tool for the matching of the RF ExB-Dipole. With a well cooled beam, a sensitivity to amplitude and phase changes in the per mill regime has been achieved.

For polarimetry runs, the vertical emittance of the deuteron beam is slowly increased and particles are gradually moved onto the carbon target, providing a stable event rate in the four quadrant polarimeter detector [8]. Beam polarization leads to an asymmetry in the angular distribution of ${ }^{12} \mathrm{C}(\overrightarrow{\mathrm{d}}, \mathrm{d})$ scattering. In case of a vertical polarized beam, it gives an asymmetry in the event rates of the left and right quadrants of the polarimeter detector. To cancel first-order contributions to systematic errors, the asymmetries were evaluated in terms of the cross ratio $C R_{\mathrm{LR}}$, combining the event rates of both the up and down polarization state [9].

The RF induced artificial spin resonance leads to a continuous, adiabatic rotation of the polarization vector, corresponding to an oscillation of its vertical component, as shown in Fig. 3. The damping of this oscillation is caused by two main sources: the decoherence of the polarization oscillation of the many particles involved [10] and the difference in particle's times of arrival at the RF ExB-Dipole. A complete spin flip occurs only if the RF device is operated exactly on the spin resonance frequency, otherwise the excitation will slip beneath the precessing spin, reducing the amplitude of the detected polarization oscillation while simultaneously increasing its frequency. This allows the determination of the spin resonance frequency down to $\approx 0.01 \mathrm{~Hz}$ with a series of runs as depicted in Fig. 3. It also constitutes a measurement of the strength of the RF induced resonance $\varepsilon=\frac{f_{P_{y}}}{f_{\text {rev }}}$.

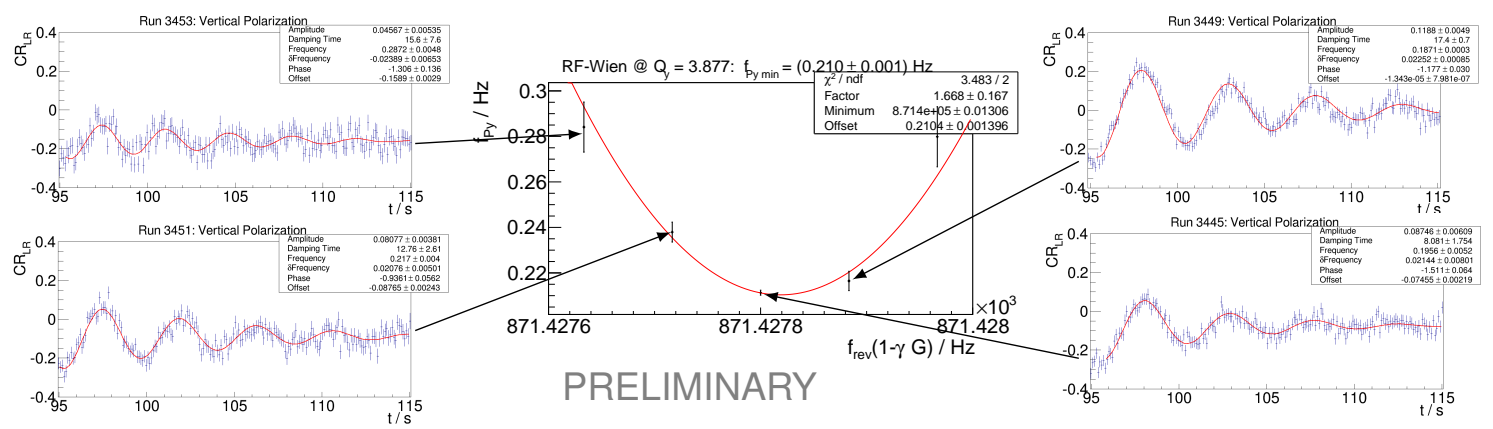

Figure 3: The left and right panels show RF driven oscillations of the vertical polarization component from a series of runs taken during the September 2014 beam-time with different excitation frequencies close to the spin resonance at $871427.80 \mathrm{~Hz}$. In the center panel, a spin resonance curve in form of excitation vs. polarization oscillation frequency is plotted. 
A series of such resonance scans have been taken during the September 2014 and May 2015 JEDI beam-times at COSY. For a range of vertical betatron tunes with sidebands around the spin resonance comparison measurements were performed between the RF ExB-Dipole in Wien-Filtermode, the RF ExB-Dipole operated without compensating electric field as a pure RF B-Dipole and with only the electric field switched on as a pure RF E-Dipole and with an already installed RF Solenoid.

Fig. 4 shows, that, like the RF Solenoid, the RF Wien-Filter doesn't excite any coherent beam oscillations. The resonance strength is independent of the vertical betatron tune. In contrast, the resonance strength of the pure RF dipoles is dominated by the interference between the driven spin motion and the one induced by coherent beam oscillations. Experimentally, this effect was already observed by experiments with resonance strength measurements by the SPIN@COSY Collaboration [11].

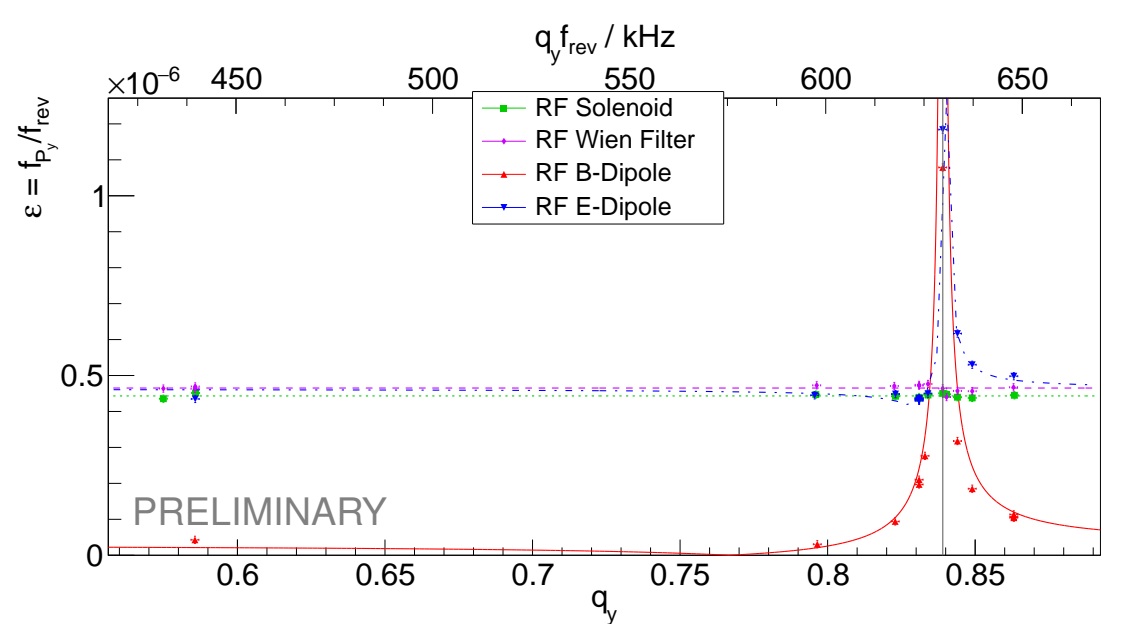

Figure 4: Results of the resonance scans at the nominal tune of COSY at $q_{y}=0.56$ and for a set of vertical betatron tunes with sideband frequencies around the spin resonance at, depicted by the vertical line at $629777.3 \mathrm{~Hz}$.

A constant fit to the measurements of the RF Wien-Filter gives a resonance strength of $\varepsilon=(4.65 \pm 0.04) \cdot 10^{-7}$ for the measured current amplitude of $\hat{I}=(0.740 \pm 0.005)$ A. The resulting normalized effective field strength of the system agrees well with the estimate from the field simulations given in Tab. 1:

$$
\int \hat{B}_{x} \mathrm{~d} l=\varepsilon \frac{4 \pi \gamma}{1+G} B \rho=(2.48 \pm 0.02) \cdot 10^{-5} \mathrm{Tm}, \quad \frac{\int \hat{B}_{x} \mathrm{~d} l}{\hat{I}}=(3.36 \pm 0.04) \cdot 10^{-5} \mathrm{Tm} / \mathrm{A} .
$$

\section{Conclusion}

As a preparation for future EDM experiments in storage rings, a first prototype of an RF WienFilter has been commissioned at COSY. We have shown, that this device generates a configuration of RF dipole fields which allow spin manipulation in a storage ring without beam disturbance. Since the cancellation of the Lorentz force depends on the particles' velocity, beam-based matching of electric and magnetic fields has been verified during the September 2014 and May 2015 JEDI beam-times at COSY. 


\section{References}

[1] V. Bargmann, Louis Michel, and V. L. Telegdi, Phys. Rev. Lett. 2, 435 (1959).

[2] T. Fukuyama and A. J. Silenko, Int. J. Mod. Phys. A 28, 1350147 (2013).

[3] W. M. Morse, Y. F. Orlov and Y. K. Semertzidis, Phys. Rev. ST Accel. Beams 16, 114001 (2013).

[4] N. N. Nikolaev, Duality of the MDM-transparent RF-E Flipper to the EDM transparent RF Wien-Filter at all Magnetic Storage Rings, EDM Note, (2012).

[5] M. Bai, W. W. MacKay, and T. Roser, Phys. Rev. ST Accel. Beams 8, 099001 (2005).

[6] S. Y. Lee, Phys. Rev. ST Accel. Beams 9, 074001 (2006).

[7] R. Maier, NIM A 390, 1-8 (1997).

[8] Z. Bagdasarian et al., Phys. Rev. ST Accel. Beams 17, 052803 (2014).

[9] G.G. Ohlsen and P.W. Keaton, Jr., NIM 109, 41-59 (1973).

[10] D. Eversmann et al., Phys. Rev. Lett. 115, 094801 (2015).

[11] A. D. Krisch, Phys. Rev. ST Accel. Beams 10, 071001 (2007). 